\title{
Consequences of coronavirus disease-2019 (COVID-19) lockdown on infection-related hospitalizations among the pediatric population in Denmark
}

\author{
Laura Bech Polcwiartek ${ }^{1}$ (D) - Christoffer Polcwiartek ${ }^{2}$. Mikkel Porsborg Andersen ${ }^{3} \cdot$ Lauge $_{\text {(stergaard }}{ }^{4}$. \\ Marcella D. Broccia ${ }^{3,5}$. Gunnar H. Gislason ${ }^{6,7}$ • Lars Køber ${ }^{4}$. Christian Torp-Pedersen ${ }^{2,3}$ • Morten Schou ${ }^{7}$. \\ Emil Fosbøl $\left.\right|^{4}$ Kristian Kragholm ${ }^{2,8,9} \cdot$ Søren Hagstrøm ${ }^{1,10,11}$
}

Received: 13 July 2020 / Revised: 30 November 2020 / Accepted: 6 January 2021 / Published online: 8 February 2021

(C) The Author(s), under exclusive licence to Springer-Verlag GmbH, DE part of Springer Nature 2021

\begin{abstract}
It remains unknown how Coronavirus disease-2019 (COVID-19) prevention measures implemented on March 12, 2020, have affected the rate of pediatric infection-related hospitalizations in Denmark. Therefore, we investigated the rate of pediatric infection-related hospitalizations during the COVID-19 pandemic. We used a retrospective cohort design and included all Danish children $<18$ years. Infection-related hospitalizations were assessed during study periods in 2020 vs. 2018/2019, and we computed incidence rate ratios (IRRs) with $95 \%$ confidence intervals (CIs) using Poisson regression. In the 2020 study period, 3093 children were hospitalized with an infection, while the corresponding figures for 2018 and 2019 study periods were 4824 and 3830, respectively. When comparing the 2020 to the 2018/2019 study period prior to nationwide lockdown, we observed a decline in infection-related hospitalizations (12.68 (95\% CI, 12.22-13.16) vs. 15.49 (95\% CI, 15.12-15.86) per 1000 personyears). We further observed decreased IRRs, especially during the lockdown period (week 11: 0.64 (95\% CI, 0.55-0.75); week 12: 0.26 (95\% CI, 0.21-0.33); week 13: 0.13 (95\% CI, 0.10-0.19)).

Conclusion: The rate of pediatric infection-related hospitalizations in Denmark declined during the COVID-19 pandemic in 2020 compared to that in 2018/2019, with a 36\% decline during initiation of the nationwide lockdown period.
\end{abstract}

\section{What is Known:}

- Due to the COVID-19 pandemic, several countries have implemented mitigation strategies such as lockdown of non-critical business functions. Most of these strategies have previously been proven effective on interruption of infection transmission.

- It remains unclear how the mitigation strategies have affected the rate of pediatric infection-related hospitalizations.

What is New:

- Insight on how COVID-19 prevention measures have affected the frequency of infection-related hospitalization.

- Valuable knowledge on how to act in potential future pandemics.

Laura Bech Polcwiartek

laurafbech@hotmail.com

Christoffer Polcwiartek

c.polcwiartek@gmail.com

Mikkel Porsborg Andersen

mikkel.porsborg.andersen@ regionh.dk

Lauge Østergaard

laugeoestergaard@gmail.com

Marcella D. Broccia

mdbro@dcm.aau.dk

Gunnar H. Gislason

gunnar.gislason@regionh.dk

\author{
Lars Køber \\ lars.koeber.01@regionh.dk \\ Christian Torp-Pedersen \\ christian.tobias.torp-pedersen@ regionh.dk \\ Morten Schou \\ morten.schou.04@ regionh.dk \\ Emil Fosbøl \\ emil.fosboel@regionh.dk \\ Kristian Kragholm \\ kdks@rn.dk \\ Søren Hagstrøm \\ soha@rn.dk
}

Extended author information available on the last page of the article 
Keywords Pediatric · Infections · COVID-19 · Epidemiology

\begin{tabular}{|c|c|}
\hline Abbreviations & \\
\hline ATC & Anatomical Therapeutic Chemical \\
\hline CIs & Confidence intervals \\
\hline COVID-19 & Coronavirus disease-2019 \\
\hline ICD-10 & $\begin{array}{l}\text { International Classification of } \\
\text { Diseases, 10th Edition }\end{array}$ \\
\hline IRRs & Incidence rate ratios \\
\hline PY & Person-years \\
\hline RSV & Respiratory syncytial virus \\
\hline SARS-CoV-2 & $\begin{array}{l}\text { Severe acute respiratory } \\
\text { syndrome coronavirus } 2\end{array}$ \\
\hline
\end{tabular}

\section{Introduction}

In the effort of containing the ongoing pandemic caused by Coronavirus disease-2019 (COVID-19), all non-critical business functions and both public and private childcare systems including nurseries, kindergarten, and schools in Denmark were locked down on March 12, 2020 [1]. Studies have reported that COVID-19 rarely requires hospitalization among children compared to adults, which is surprising considering that infections are the main cause of morbidity and mortality among children and account for approximately $80 \%$ of all pediatric hospitalizations [2-6]. However, evidence from prior influenza outbreaks suggests that children may play an important role in the transmission of COVID-19, thus underscoring the importance of educating children and parents on social distancing and proper hygiene measures, which are recognized factors in prevention of infection transmission [7-9]. Considering the limited literature on how such largescale mitigation strategies may affect various infections, it remains unknown if the rate of infection-related hospitalizations among children has been affected [6,9]. Together with the increased focus on hygiene and social distancing, the fear of contracting COVID-19 may play a role in lowering hospitalization rates in that parents may refrain from seeking medical care despite having an ill child, as hypothesized by others [10-12]. On the contrary, mass media reports on the ongoing pandemic may induce negative behavior in some parents causing them to lower their threshold for seeking medical care [13]. Likewise, it could be speculated that clinicians may tend to hospitalize more children for observation than they usually do in fear of missing severe infections.

Therefore, we aimed to investigate the rate of infection-related hospitalizations in the Danish pediatric population during the COVID-19 pandemic using Danish nationwide register data. As a control, we also investigated the rate of epilepsy-related hospitalizations, which is one of the most frequent chronic diseases among children, and we hypothesized that it would not be affected by the COVID-19 prevention measures.

\section{Material and methods}

\section{Study design, population, and period}

We conducted a nationwide register-based retrospective cohort study including all children $<18$ years in Denmark who were alive at index date (January 2, 2018, January 2, 2019, and January 2, 2020) and were hospitalized due to an infection.

\section{Registers, covariates, and definitions}

At birth or time of immigration, all Danish citizens are assigned a unique personal identification number registered in the Danish Civil Population Register [14], which is used in all healthcare contacts facilitating confidential linkage between registers in Denmark. From this register, we included data on date of birth and sex. Data on vital status was obtained from the Danish Register of Causes of Death [15]. International Classification of Diseases, 10th Edition (ICD-10) codes from all infection- and epilepsy-related hospitalizations were retrieved from the Danish National Patient Register [16]. From the Danish National Prescription Register [17] containing data on filled prescriptions from all Danish pharmacies since 1995, we obtained data on filled drug prescriptions based on Anatomical Therapeutic Chemical (ATC) codes.

Using ICD-10 codes, we identified comorbidities including asthma, diabetes, urinary incontinence, chronic kidney disease, liver disease, rheumatic disease, and malignancy diagnosed prior to index date. Using ATC codes, we identified filled prescriptions for antidiabetics, drugs for urinary incontinence, inhalants for obstructive airway disease, and antiinflammatory drugs such as corticosteroids within 90 days prior to the index date. Furthermore, we identified filled prescriptions for antibiotics within 7 days prior to index date. Children filling prescriptions for antidiabetics or drugs for urinary incontinence were defined as having diabetes and urinary incontinence accordingly (see Supplementary eTable 1 for a complete list of ICD-10 and ATC codes).

We compared data for three overall and one lockdown study period. The three overall study periods comprised January 2, 2018-April 3, 2018; January 2, 2019-April 3, 2019; and January 2, 2020-April 2, 2020. The lockdown study period was from March 12, 2020 to April 2, 2020, and the comparison periods were from March 12, 2018 to April 2, 2018 and March 12, 2019 to April 2, 2019. 
We defined infection-related hospitalizations as children admitted to the hospital due to an infection. Children, who were hospitalized due to another cause and developed an infection during the hospitalization, were not included.

\section{Outcome}

Our main outcome was infection-related hospitalizations lasting more than $24 \mathrm{~h}$. We included systemic, central nervous system, ear, respiratory, gastrointestinal, urological, and skin infections. Respiratory-related hospitalizations were further stratified by the type of agent including influenza virus, respiratory syncytial virus (RSV), COVID-19, bacterial, and other unspecified agents. In addition, we investigated epilepsyrelated hospitalizations lasting more than $24 \mathrm{~h}$ during the same study periods. We included this chronic disease as a control, because we hypothesized that the rate of hospitalizations due to epilepsy would not change during the COVID-19 pandemic as opposed to infections. Finally, to ensure that a change in infection-related hospitalization rates was not driven by allcause mortality, we investigated this as a secondary outcome (see Supplementary eTable 2 for a complete list of ICD-10 codes). Children were followed from the index date to the date of hospitalizations, date of death, or date of exit (April 3, 2018; April 3, 2019; or April 2, 2020).

\section{Definition of data and statistical analysis}

Categorical variables were reported as counts with corresponding percentages and continuous variables as medians with 25th and 75th percentiles. To maintain confidentiality, observations $<3$ will be reported as $<3$ and not as actual numbers.

First, we reported baseline characteristics of children hospitalized due to an infection as well as the number of first-time infection-related hospitalizations by weeks during the study periods. Here, children could only be included once per study period, but could appear in multiple study periods; e.g., a child could be hospitalized with an infection in 2019 and in 2020 . Second, we reported the number of infection-related hospitalizations by infection type and weeks counting from the index date during the study periods. Here, children could only be included once per ICD-10 code in each study period, but could appear with multiple infection types and in multiple study periods; e.g. a child could be hospitalized with an RSV infection in 2019 and later an influenza infection in 2019. Third, we estimated rates for the first 10 weeks prior to nationwide lockdown and for the 3 weeks during nationwide lockdown for 2018/2019 and 2020 study periods. In addition, using Poisson regression analysis, we computed crude incidence rate ratios (IRRs) with $95 \%$ confidence intervals (CIs) for each week during the 2020 study period compared to that during the 2018/2019 study period, which served as reference. Finally, we calculated the rate of all-cause mortality for weeks $1-10$ and 11-13 during the study periods.

As subanalyses, we calculated crude IRRs for all infectionrelated hospitalizations stratified according to age $<6$ years and $\geq 6$ years. We chose these age groups because children start school at age 6 years in Denmark. Furthermore, we reported the number of children with a first-time epilepsy-related hospitalization during the study periods, and using Poisson regression analysis, we computed IRRs with $95 \%$ CIs for each week during the 2020 study period compared to that during the 2018/2019 study period, which served as reference.

Data management was conducted using SAS version 9.4 (SAS Institute, Inc., Cary, NC, USA) and data analysis using $\mathrm{R}$ version 3.6.1 (R Foundation for Statistical Computing, Vienna, Austria).

\section{Ethics}

In Denmark, register-based studies do not require ethical committee approval or patient consent. Approval to use the data sources for research purposes was granted by the data responsible institute in the Capital Region of Denmark in accordance with the General Data Protection Regulation (GDPR) approval number P-2019-191.

\section{Results}

\section{Patients and characteristics}

During the 2018, 2019, and 2020 study periods, we included $1,147,884 ; 1,142,993$; and $1,139,333$ children, respectively (Table 1). Overall, the baseline characteristics of children hospitalized due to an infection during the study periods were similar, with a median age of approximately 2 years and the majority being male. In the 2018 study period, 4824 children were hospitalized with an infection, where $6 \%$ suffered from asthma, $14 \%$ filled prescriptions for inhalants for obstructive airway disease, and $9 \%$ filled prescriptions for antibiotics. Correspondingly, of the 3830 children hospitalizations during the 2019 study period, $6 \%$ suffered from asthma, $11 \%$ filled prescriptions for inhalants for obstructive airway disease, and 9\% filled prescriptions for antibiotics. Finally, of the 3093 children hospitalized during the 2020 study period, $6 \%$ suffered from asthma, $11 \%$ filled prescriptions for inhalants for obstructive airway disease, and $3 \%$ filled prescriptions for antibiotics.

\section{Infection-related hospitalizations}

Among the 3093 children hospitalized in the 2020 study period, we identified 3241 infection-related hospitalizations, while the corresponding figures for 2018 and 2019 study 
Table 1 Baseline characteristics of children hospitalized due to an infection during the three study periods

\begin{tabular}{llll}
\hline \multirow{2}{*}{ Characteristics } & \multicolumn{3}{l}{ Overall study period (January-April) } \\
\cline { 2 - 4 } & 2018 & 2019 & 2020 \\
\hline Danish pediatric population & $1,147,884$ & $1,142,993$ & $1,139,333$ \\
Number of first-time hospitalizations & 4824 & 3830 & 3093 \\
Age, years & $1.6(0.7,5.5)$ & $1.5(0.8,3.0)$ & $1.5(0.7,3.9)$ \\
Males & $2562(53.1)$ & $2171(56.7)$ & $1775(57.4)$ \\
Asthma & $290(6.0)$ & $211(5.5)$ & $171(5.5)$ \\
Diabetes & $19(0.4)$ & $17(0.4)$ & $12(0.4)$ \\
Urine incontinence & $49(1.0)$ & $6(0.2)$ & $16(0.5)$ \\
Chronic kidney disease & $87(1.8)$ & $37(1.0)$ & $33(1.1)$ \\
Liver disease & $13(0.3)$ & $11(0.3)$ & $13(0.4)$ \\
Rheumatic disease & $45(0.9)$ & $19(0.5)$ & $16(0.5)$ \\
Malignancy & $26(0.5)$ & $24(0.6)$ & $19(0.6)$ \\
Inhalants for obstructive airway disease & $684(14.2)$ & $427(11.1)$ & $353(11.4)$ \\
Anti-inflammatory drugs & $145(3.0)$ & $83(2.2)$ & $55(1.8)$ \\
Antibiotics & $442(9.2)$ & $327(8.5)$ & $102(3.3)$ \\
\hline
\end{tabular}

periods were 5058 among 4824 children and 4007 among 3830 children, respectively (Table 2). Overall, the number of infection-related hospitalizations declined in the 2020 study period relative to 2018 and 2019 study periods (Fig. 1a).

Table 2 Characteristics of infection type during the three study periods

\begin{tabular}{|c|c|c|c|}
\hline Infection type & $2018(n=5058)$ & $2019(n=4007)$ & $2020(n=3241)$ \\
\hline \multicolumn{4}{|c|}{ Overall study period (January-April) } \\
\hline Respiratory & $3127(61.8)$ & $2712(67.7)$ & $2560(79.7)$ \\
\hline Influenza virus & $175(5.6)$ & $232(8.6)$ & $241(9.4)$ \\
\hline RSV & $741(23.7)$ & $516(19.0)$ & $473(18.5)$ \\
\hline SARS-CoV-2 & $0(0.0)$ & $0(0.0)$ & $14(0.5)$ \\
\hline Bacterial & $144(4.6)$ & $102(3.8)$ & $66(2.6)$ \\
\hline Unspecified & $2067(66.1)$ & $1862(68.7)$ & $1766(69.0)$ \\
\hline Gastrointestinal & $871(17.2)$ & $1121(28.0)$ & $477(14.8)$ \\
\hline \multirow[t]{2}{*}{ Other $^{\mathrm{A}}$} & $1060(21.0)$ & $174(4.3)$ & $177(5.5)$ \\
\hline & $2018(n=1065)$ & $2019(n=1143)$ & $2020(n=380)$ \\
\hline \multicolumn{4}{|c|}{ Lockdown period (March-April) } \\
\hline Respiratory & $576(54.1)$ & $613(53.6)$ & $285(75.0)$ \\
\hline Influenza virus & $50(8.7)$ & $18(2.9)$ & $34(11.9)$ \\
\hline RSV & $93(16.1)$ & $107(17.5)$ & $32(11.2)$ \\
\hline SARS-CoV-2 & $0(0.0)$ & $0(0.0)$ & $4(1.4)$ \\
\hline Bacterial & $26(4.5)$ & $23(3.8)$ & $10(3.5)$ \\
\hline Unspecified & 407 (70.7) & 465 (75.9) & 205 (71.9) \\
\hline Gastrointestinal & $246(23.1)$ & 489 (42.8) & $59(15.5)$ \\
\hline Other ${ }^{\mathrm{A}}$ & $243(22.8)$ & $41(3.6)$ & $36(9.5)$ \\
\hline
\end{tabular}

Abbreviations: $R S V$ respiratory syncytial virus, $S A R S-C o V-2$ severe acute respiratory syndrome coronavirus 2

${ }^{A}$ Including central nervous system, ear, skin, and urological infections
Correspondingly, respiratory infections that were the most frequent cause of hospitalization during all three study periods declined in the 2020 study period. The most pronounced decline occurred after week 11, where the nationwide lockdown period was initiated (Fig. 1b). A less pronounced decline was observed for gastrointestinal infections (Fig. 1c).

Among all infection-related hospitalizations in the 2020 study period, respiratory and gastrointestinal infections comprised a total of $80 \%$ and $15 \%$ of the hospitalizations, respectively (Table 2). In the 2019 study period, respiratory and gastrointestinal infections comprised a total of $68 \%$ and $28 \%$ of the hospitalizations, respectively. In 2018, respiratory and gastrointestinal infections comprised a total of $62 \%$ and $17 \%$ of the hospitalizations, respectively. Of recognized respiratory infection agents, RSV was the most frequent during all study periods, whereas 14 hospitalizations were due to COVID-19 infections in 2020 (Table 2).

\section{Comparison of the rate of infection-related hospitalizations}

Prior to the nationwide lockdown (weeks 1-10) in 2020, the rate of infection-related hospitalizations was 12.68 (95\% CI, 12.22-13.16) per 1000 person-years (PY). During the 2020 lockdown period (week 11-13), the rate declined substantially to 5.01 (95\% CI, 4.49-5.58) per 1000 PY. In the 2018/2019 study period, no substantial decline was observed (prior to: 15.49, 95\% CI, $15.12-15.86$ per 1000 PY; during: 14.21 ; $95 \%$ CI, 13.58-14.87 per 1000 PY).

Compared to the 2018/2019 study period, we observed a significant decrease in IRRs from week 4 in the 2020 study 
a First-Time Infection-Related Hospitalization

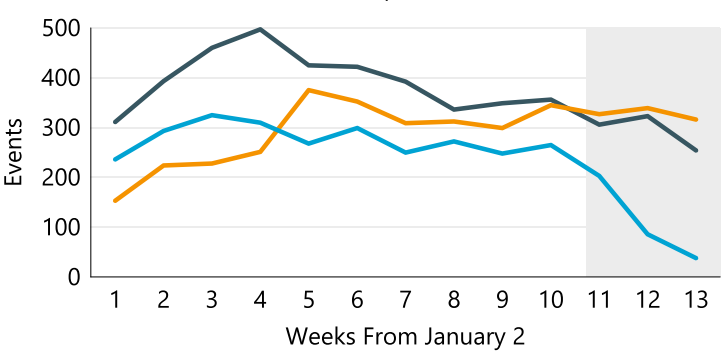

C Gastrointestinal Infection-Related Hospitalizations

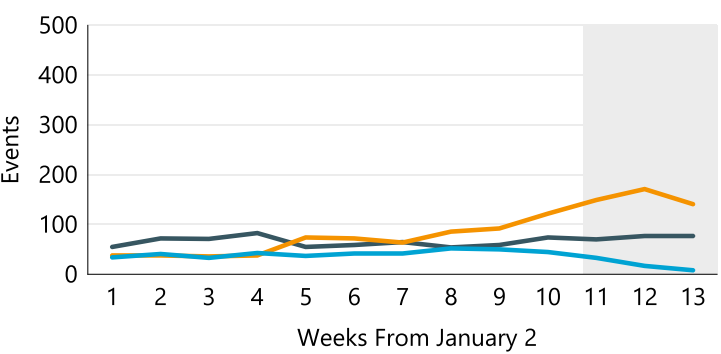

b Respiratory Infection-Related Hospitalizations

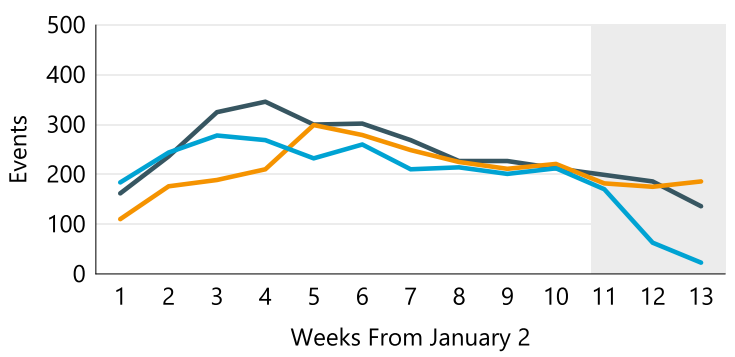

$$
\begin{gathered}
2018-2019-2020 \\
\text { Nationwide Lockdown }
\end{gathered}
$$

Fig. 1 Rates of infection-related hospitalizations, where children can appear only once per study period (a), respiratory infection-related hospitalizations, where children can appear multiple time per infection

period, with the most pronounced decrease during the lockdown period (week 11: $0.64,95 \%$ CI, $0.55-0.75$; week 12: 0.26, 95\% CI, 0.21-0.33; week 13: 0.13, 95\% CI, 0.10-0.19)

(Fig. 2).

For baseline characteristics during the lockdown study periods, see Supplementary eTable 3.

\section{Rate of all-cause mortality}

In the 2020 study period, no difference was observed when comparing all-cause mortality rates prior to lockdown $(0.10$, 95\% CI, 0.07-0.15 per $1000 \mathrm{PY})$ and during lockdown (0.15, 95\% CI, 0.08-0.28 per 1000 PY). In the 2018/2019 study period, similar trends were observed (weeks 1-10: 0.14, 95\% CI, 0.11-0.18 per 1000 PY; weeks 11-13: 0.16, 95\% CI, $0.10-0.25$ per $1000 \mathrm{PY}$ )

\section{Subanalyses}

When stratifying infection-related hospitalizations by age $<6$ or $\geq 6$ years, we observed similar trends as in the main analysis (see Fig. S1 in the Supplementary information).

As a measure of hospitalizations due to chronic diseases, we identified a total of 252, 319, and 293 children being hospitalized due to epilepsy in the 2018, 2019, and 2020 study periods, respectively. Compared to the 2018/2019 study period, we did not observe any differences in IRRs except for in type (b), and gastrointestinal infection-related hospitalizations, where children can appear multiple time per infection type (c) by weeks stratified by years

week 12 in the 2020 study period $(0.21,95 \%$ CI, 0.08-0.59) (see Fig. S3 in the Supplementary information).

\section{Discussion}

In this study, we found that less children were hospitalized due to an infection during the 2020 study period compared to the 2018 and 2019 study periods. Baseline characteristics for children hospitalized due to an infection were similar, with a median age of approximately 2 years and the majority being male. Furthermore, the rates of infection-related hospitalizations were lower during the 2020 study period compared to the other study periods. Especially, after week 11 in the 2020 study period, in which the nationwide lockdown period was initiated, the infection rate decreased by $36 \%$. Respiratory infections were the most frequent cause of hospitalization, with RSV being the most frequent agent. Of note, all-cause mortality rate was similar during the study periods, and no increase was observed when comparing rates prior to and during the nationwide lockdown period.

Similar to our findings, other studies have reported a decrease in the rate of infection-related hospitalizations after the initiation of lockdown [6]. Our findings support that a nationwide lockdown may interrupt infection transmission, not only for COVID-19 but also for other severe infections [6, 18]. However, our study design did not allow for analysis of 
Fig. 2 Incidence rate ratios for infection-related hospitalizations for each week during the 2020 study period compared to that during the 2018/2019 study period. Abbreviations: CI confidence interval, IRR incidence rate ratio

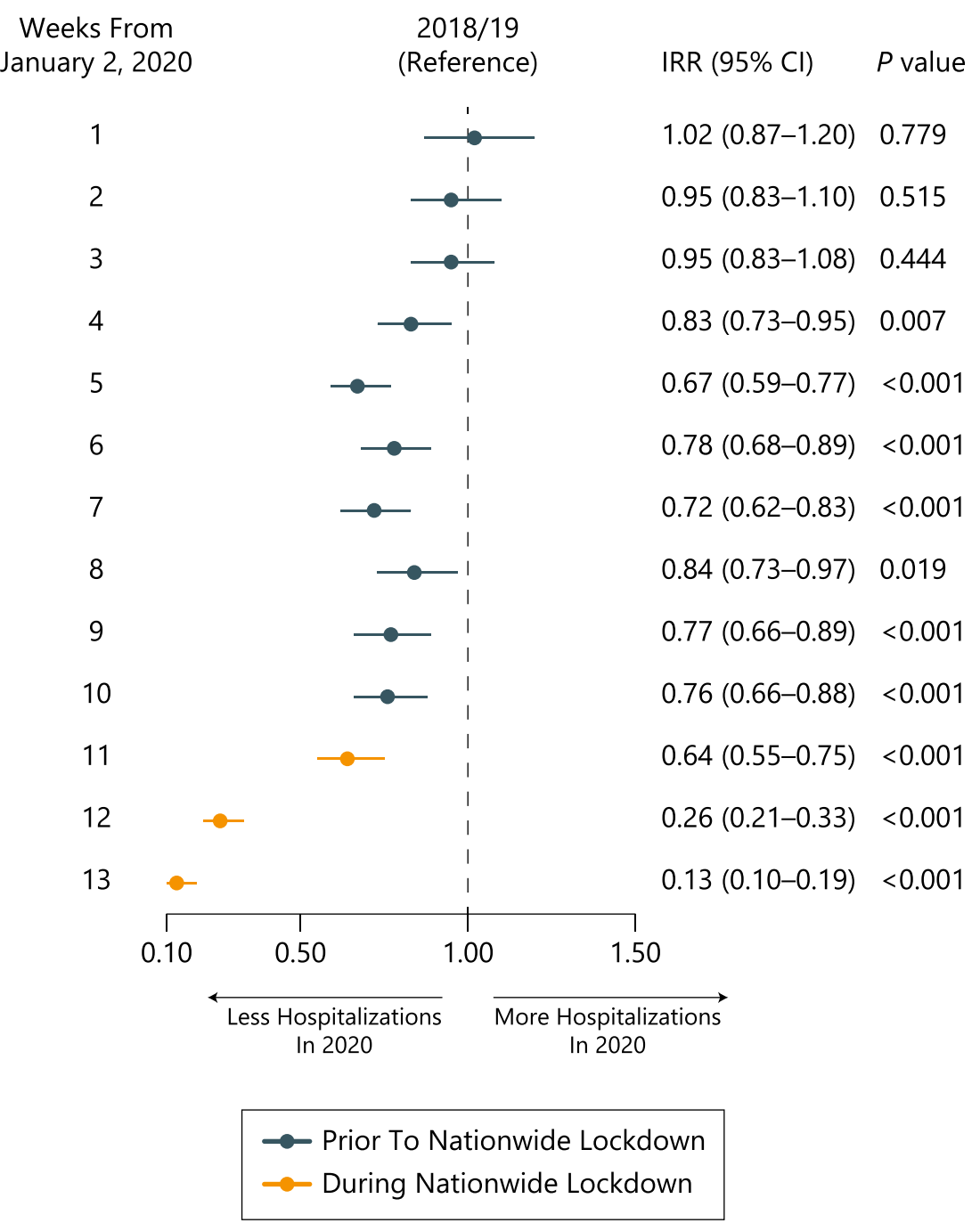

possible negative secondary consequences of COVID-19 prevention measures such as school closure and social distancing as suggested in other studies [7, 19-21]. Of note, the frequency of hospitalized children with comorbidities did not appear to differ between study periods. In these times, this is an important notion, as parents of children with chronic diseases such as asthma tend to show concerns and anxiety on whether their child may be more prone to contract severe COVID-19 infection requiring hospitalization.

Because epilepsy is another common cause of hospitalization among children, we used this as a proxy measure to investigate whether chronic diseases may also have been affected by the COVID-19 prevention measures. However, we did not find a similar downward trend in hospitalization rates when comparing the study periods. Although this was not surprising, the fear of contracting COVID-19 infection could have refrained parents from seeking medical care and managed seizures at home.

We investigated both highly transmissible and seasonal infections such as respiratory and gastrointestinal infections as well as infections less likely to be transmissible such as skin and urological infections. As hypothesized, respiratory and gastrointestinal infection-related hospitalizations decreased substantially during the 2020 study period compared to those during the 2018 and 2019 study periods. However, it was surprising that the distribution of infection type during the 2020 study period was skewed toward respiratory considering findings from three recent studies reporting that respiratory infections were significantly lower during the 2019/2020 season compared to the $2018 / 2019$ season $[6,9,22]$. The latter is supported by a recent report from the Danish Heath Authorities [23].

Among the 3241 infection-related hospitalizations in the 2020 study period, we identified 14 COVID-19 infections. In the context of the COVID-19 pandemic, prior studies stated that COVID-19 and influenza virus may have similar transmission mode and virulence causing speculations on whether influenza virus activity may be used as a proxy for COVID-19 surveillance [6,9]. Considering that COVID-19-infected children rarely develop severe illness requiring hospitalization 
together with the fact that we only investigated infections requiring hospitalization, our study design did not allow us to explore whether the rate of COVID-19 infections in children decreased during the 2020 study period, particularly during the nationwide lockdown period [4, 24]. However, based on advice from the Danish Health Authorities, the Danish government initiated a plan for a graduate reopening of the society such as nurseries, kindergarten, and primary schools on April 14, 2020, in parallel with flattening of the COVID-19 infection curve [1].

Although interruption of the infection transmission caused by the nationwide lockdown is an important factor in explaining our findings, several other factors may also have an important role. Clinicians may have improved their ability to determine which patients require urgent hospitalization and which can be managed as outpatients. In addition, parents may avoid seeking medical care due to the risk of contracting COVID-19 infection or because they simply do not want to overburden the healthcare system $[10,11]$. However, as the mortality rate did not differ substantially between the study periods, it seems less likely that parents refrained from seeking medical care if their child was severely ill. Secondary to reduce the amount of acute infections requiring hospitalization and thereby not overburdening the healthcare system, the COVID-19 prevention measures may also have positive long-term implications in that acute infections predispose children to chronic diseases [25]. Thus, by educating children in proper hygienic measures and social distancing, it is possible that the incidence of chronic diseases among some children will decline. Further studies exploring the role of various prevention efforts such as nationwide lockdown during the COVID-19 pandemic on other pediatric conditions are warranted [7]. Finally, considering that baseline characteristics such as median age, sex, and comorbidities were similar across study periods, it seems less likely that these factors have influenced the number of infectionrelated hospitalizations.

Our study was limited by its observational design in that unmeasured confounding factors may have affected findings. Our registers do not contain data on clinical signs and symptoms, blood tests, or cultures. Although vaccines for the most common and severe pediatric infections are part of the free childhood immunization schedule in Denmark, they are not mandatory, and we did not have access to data on whether children had received all the recommended vaccines. Only children hospitalized for a minimum of $24 \mathrm{~h}$ were included, and often, children are hospitalized for shorter periods or treated and observed in the emergency department. This may underestimate the rate of infections and epilepsy potentially requiring hospitalization to a pediatric department in our study. Furthermore, due to the sample size, we were not able to stratify according to age groups. Finally, we had only access to data from the acute phase of the COVID-19 pandemic. As such, knowledge on long-term consequences on the healthcare system of the COVID-19 pandemic remains unclear [26]. In conclusion, the rate of all infection-related hospitalizations among children in Denmark declined during the COVID-19 pandemic in 2020 compared to that in 2018/2019, with a decline of $36 \%$ during initiation of the nationwide lockdown period. In contrast, the rate of epilepsy-related hospitalizations did not differ when comparing the study periods. This indicates that although some parents may refrain from seeking medical care due to the fear of contracting COVID-19 infection, proper hygiene measure and social distancing could have positive secondary consequences on the rate of other infections among the pediatric population.

Supplementary Information The online version contains supplementary material available at https://doi.org/10.1007/s00431-021-03934-2.

Authors' contributions LBP: conceptualization, methodology, formal analysis and investigation, drafting of manuscript and manuscript preparation.

CP: methodology, formal analysis and investigation, manuscript revision.

MPA: manuscript revision.

LØ: formal analysis and investigation, and manuscript revision.

MDB: manuscript revision.

GHG: manuscript revision, resources, and supervision.

LK: manuscript revision, resources, and supervision.

CT-P: manuscript revision, resources, and supervision.

MS: manuscript revision, resources, and supervision.

EF: manuscript revision, resources, and supervision.

KK: conceptualization, mythology, manuscript revision, and supervision.

$\mathrm{SH}$ : manuscript revision, funding acquisition, and supervision.

\section{Compliance with ethical standards}

Conflict of interest $\mathrm{CP}$ reported receiving speaking fees from Lundbeck Pharma A/S and research grants from the Danish Heart Foundation and the Eva and Henry Frænkel Memorial Foundation. GHG reported receiving research grants from Bristol-Myers Squibb, Pfizer, Boehringer Ingelheim, and Bayer. LK reported receiving lecture fees from Novartis and Bristol-Myers Squibb. CT-P reported receiving research grants from Bayer. EF reported receiving independent research grant from Janssen Pharmaceutical and BMS. KK reported receiving speaking fees from Novartis and research grants from the Laerdal Foundation. LBP, MPA, LØ, MDB, MS, and SH declare that they have no conflict of interest.

Ethical approval This article does not contain any studies with human participants or animals performed by any of the authors. Approval to use the data sources for research purposes was granted by the data responsible institute in the Capital Region of Denmark in accordance with the General Data Protection Regulation (GDPR) approval number P-2019-191.

Consent to participate N/A

Consent for publication N/A

Data availability N/A

Code availability N/A 


\section{References}

1. Danish Health Authority. Tal og overvågning af COVID-19.2020.

2. Dossetor PJ, Martiniuk ALC, Fitzpatrick JP, Oscar J, Carter M, Watkins R, Elliott EJ, Jeffery HE, Harley D (2017) Pediatric hospital admissions in indigenous children: a population-based study in remote Australia. BMC Pediatr 17:195

3. Paret M, Lighter J, Pellett Madan R, Raabe VN, Shust GF, Ratner AJ (2020) SARS-CoV-2 infection (COVID-19) in febrile infants without respiratory distress. Clin Infect Dis

4. Team CC-R (2020) Coronavirus disease 2019 in children - United States, February 12-April 2, 2020. MMWR Morb Mortal Wkly Rep 69:422-426

5. Li Q, Guan X, Wu P, Wang X, Zhou L, Tong Y, Ren R, Leung KSM, Lau EHY, Wong JY, Xing X, Xiang N, Wu Y, Li C, Chen Q, Li D, Liu T, Zhao J, Liu M, Tu W, Chen C, Jin L, Yang R, Wang Q, Zhou S, Wang R, Liu H, Luo Y, Liu Y, Shao G, Li H, Tao Z, Yang Y, Deng Z, Liu B, Ma Z, Zhang Y, Shi G, Lam TTY, Wu JT, Gao GF, Cowling BJ, Yang B, Leung GM, Feng Z (2020) Early transmission dynamics in Wuhan, China, of novel coronavirus-infected pneumonia. N Engl J Med 382:1199-1207

6. Cowling BJ, Ali ST, Ng TWY, Tsang TK, Li JCM, Fong MW, Liao Q, Kwan MY, Lee SL, Chiu SS, Wu JT, Wu P, Leung GM (2020) Impact assessment of non-pharmaceutical interventions against coronavirus disease 2019 and influenza in Hong Kong: an observational study. Lancet Public Health 5:e279-e288

7. Viner RM, Russell SJ, Croker H, Packer J, Ward J, Stansfield C, Mytton O, Bonell C, Booy R (2020) School closure and management practices during coronavirus outbreaks including COVID-19: a rapid systematic review. Lancet Child Adolesc Health 4:397-404

8. Jackson C, Vynnycky E, Mangtani P (2016) The relationship between school holidays and transmission of influenza in England and Wales. Am J Epidemiol 184:644-651

9. Zipfel CM, Bansal S (2020) Assessing the interactions between COVID-19 and influenza in the United States. medRxiv

10. Sen-Crowe B, McKenney M, Elkbuli A (2020) Social distancing during the COVID-19 pandemic: staying home save lives. Am J Emerg Med 38:1519-1520

11. Patrick K, Stanbrook MB, Laupacis A (2020) Social distancing to combat COVID-19: We are all on the front line. CMAJ : Can Med Assoc J 192:E516-E517

12. Birkmeyer JD, Barnato A, Birkmeyer N, Bessler R, Skinner J (2020) The impact of the COVID-19 pandemic on hospital admissions in the United States. Health Aff 39:2010-2017

13. Collinson S, Khan K, Heffernan JM (2015) The effects of media reports on disease spread and important public health measurements. PLoS One 10:e0141423

14. Pedersen CB (2011) The Danish Civil Registration System. Scand J Public Health 39:22-25
15. Helweg-Larsen K (2011) The Danish Register of Causes of Death. Scand J Public Health 39:26-29

16. Lynge E, Sandegaard JL, Rebolj M (2011) The Danish National Patient Register. Scand J Public Health 39:30-33

17. Kildemoes HW, Sorensen HT, Hallas J (2011) The Danish National Prescription Registry. Scand J Public Health 39:38-41

18. Nussbaumer-Streit B, Mayr V, Dobrescu AI, Chapman A, Persad E, Klerings I, Wagner G, Siebert U, Christof C, Zachariah C, Gartlehner G (2020) Quarantine alone or in combination with other public health measures to control COVID-19: a rapid review. Cochrane Database Syst Rev 4:CD013574

19. Nicola M, Alsafi Z, Sohrabi C, Kerwan A, Al-Jabir A, Iosifidis C, Agha M, Agha R (2020) The socio-economic implications of the coronavirus pandemic (COVID-19): a review. Int J Surg 78:185193

20. Golberstein E, Wen H, Miller BF (2020) Coronavirus disease 2019 (COVID-19) and mental health for children and adolescents. JAMA Pediatr 174:819-820

21. Xie X, Xue Q, Zhou Y, Zhu K, Liu Q, Zhang J, Song R (2020) Mental health status among children in home confinement during the coronavirus disease 2019 outbreak in Hubei Province, China. JAMA Pediatr 174:898-900

22. Sakamoto H, Ishikane M, Ueda P (2020) Seasonal influenza activity during the SARS-CoV-2 outbreak in Japan. Jama 323:19691971

23. Statens Serums Institut. Influenza - ugens opgørelse. 2020. Statens Serums Institut

24. Garg S, Kim L, Whitaker M, O'Halloran A, Cummings C, Holstein R, Prill M, Chai SJ, Kirley PD, Alden NB, Kawasaki B, YouseyHindes K, Niccolai L, Anderson EJ, Openo KP, Weigel A, Monroe ML, Ryan P, Henderson J, Kim S, Como-Sabetti K, Lynfield R, Sosin D, Torres S, Muse A, Bennett NM, Billing L, Sutton M, West N, Schaffner W, Talbot HK, Aquino C, George A, Budd A, Brammer L, Langley G, Hall AJ, Fry A (2020) Hospitalization rates and characteristics of patients hospitalized with laboratoryconfirmed coronavirus disease 2019 - COVID-NET, 14 States, March 1-30, 2020. MMWR Morb Mortal Wkly Rep 69:458-464

25. O'Connor SM, Taylor CE, Hughes JM (2006) Emerging infectious determinants of chronic diseases. Emerg Infect Dis 12:1051-1057

26. Fontanarosa PB, Bauchner H (2020) COVID-19-looking beyond tomorrow for health care and society. Jama 323:1907-1908

Publisher's note Springer Nature remains neutral with regard to jurisdictional claims in published maps and institutional affiliations. 


\section{Affiliations}

Laura Bech Polcwiartek ${ }^{1}$ (1) - Christoffer Polcwiartek ${ }^{2} \cdot$ Mikkel Porsborg Andersen $^{3} \cdot$ Lauge $_{\text {(stergaard }}{ }^{\text {. }}$ Marcella D. Broccia ${ }^{3,5}$. Gunnar H. Gislason ${ }^{6,7}$ • Lars Køber ${ }^{4}$. Christian Torp-Pedersen ${ }^{2,3}$ • Morten Schou ${ }^{7}$. Emil Fosbøl ${ }^{4} \cdot$ Kristian Kragholm $^{2,8,9} \cdot$ Søren Hagstrøm ${ }^{1,10,11}$

1 Department of Pediatrics, Aalborg University Hospital, Reberbansgade 15, 9000 Aalborg, Denmark

2 Department of Cardiology, Aalborg University Hospital, Hobrovej 18-22, 9000 Aalborg, Denmark

3 Department of Clinical Research, Nordsjaellands Hospital, Dyrehavevej 29, 3400 Hillerød, Denmark

4 Department of Cardiology, Rigshospitalet, Blegdamsvej 9, 2100 Copenhagen, Denmark

5 Department of Obstetrics and Gynecology, Aalborg University Hospital, Hobrovej 18-22, 9000 Aalborg, Denmark

6 The Danish Heart Foundation, Vognmagergade 7, 3. sal, 1120 Copenhagen, Denmark
7 Department of Cardiology, Copenhagen University Hospital, Herlev and Gentofte, Gentofte Hospitalsvej 1, 2900 Hellerup, Denmark

8 Department of Cardiology, North Denmark Regional Hospital, Bispensgade 37, 9800 Hjørring, Denmark

9 Unit of Clinical Biostatistics and Epidemiology, Aalborg University Hospital, Hobrovej 18-22, 9000 Aalborg, Denmark

10 Center for Clinical Research, North Denmark Regional Hospital, Bispensgade 37, 9800 Hjørring, Denmark

11 Department of Clinical Medicine, Aalborg University, Hobrovej 18-22, 9000 Aalborg, Denmark 